\title{
Railway Signal Control Based on Intelligent Technology
}

\author{
Yu Xiao ${ }^{1, a}$ Qian Hua ${ }^{2, b}$ \\ ${ }^{1,2}$ Institute of communications signal research, China Academy of Railway \\ Science,BeiJing, 100081, China \\ a,bsnowow@126.com
}

Keywords: intelligent algorithm, railway signal, control, fault diagnosis

\begin{abstract}
C4.5 by combining with characteristics of railway signal system in China, meanwhile, it makes research and design on inference machine, explanation mechanism and human interface in expert system of fault diagnosis. It puts forward knowledge representation based on decision tree and application model of acquisition method, designs the complicated fault diagnosis algorithm based on decision tree. Through one fault case, this paper makes demonstration on effect of main modules in fault diagnosis for the designed expert system on fault diagnosis.
\end{abstract}

\section{Introduction}

Because computer interlock system of railway traffic signal not only should have continual and stable operation without stop, but also it must guarantee safety control for signal, which needs system to have higher safety and reliability, otherwise once system has fault and output is wrongly controlled, which will cause signal display update, it is possible to result in serious train operation accident as well as losses in personal and property. With the development of microelectronic technique, computer technology, automatic control technology, embedded technology and communication technology, which provides technology support for realization of railway signal fault and safety technology, full electronic computer interlock system based on high-reliability and error-tolerant safety computer is the development direction ${ }^{[4,5]}$ for interlock control technology both in China and abroad. At present, railway signal in China uses real-time state and information of micro-computer monitoring system and collection equipment, it provides reliable base to learn about operate state of equipment and analyze accident cause. However, when signal equipment has fault, railway signal system is lacking one comprehensive monitoring software synthesizes state monitoring and fault diagnosis all in one. Therefore, establishment on expert system in railway signal and equipment fault diagnosis has very realistic meanings for fault positioning and formulation of maintenance plan.

This paper designs construction model of data base based on learning algorithm of C4.5 by combining with characteristics of railway system in China; meanwhile, it makes research and design on inference machine, explanation mechanism and human interface in expert system of fault diagnosis. Finally, through one fault case, this paper makes demonstration on effect of main modules in fault diagnosis for the designed expert system on fault diagnosis. In the demonstration stage, it designs each function sub-module in expert system and realizes fault diagnosis on railway signal equipment. The test result indicates that this method can realize automatic acquisition on knowledge, which paves foundation for development of real application system.

\section{Full electronic interlock system frame based on intelligent}

The system structure of full electronic computer interlock is divided into 3 layers: operating presentation layer, safety interlock logic layer, full electronic execution layer. Operating presentation layer and safety interlock logic layer make communication by double-industry Ethernet. Safety interlock logic and full electronic execution layer make communication by optical network of ring or star type, full electronic execution layer adopts redundant CAN bus to make communication. 
Considering from safety perspective, the whole system is divided into safety region and dangerous region, of which operating presentation layer is dangerous region, while safety interlock logic layer and full electronic execution layer belong to safety region. Human interface of operating presentation layer is to mainly realize operation, presentation, maintenance of the whole system, safety interlock logic layer is based on safety interlock computer to realize each kind of interlock logic calculation, synchronization and control, each kind of electronic execution unit in full electronic execution layer realizes control output and monitoring on essential equipment of outdoor signal. Diagram 1 is the system structure diagram of full electronic computer interlock system.

In order to guarantee maintenance each sub-system in the system, each layer and function unit of system can guarantee independence with other parts in design, function, data and fault all have undergone restrict definition and package, on change of sub-system or it has external disturbance, which can effectively shield disturbance or hit for normal operation of system.

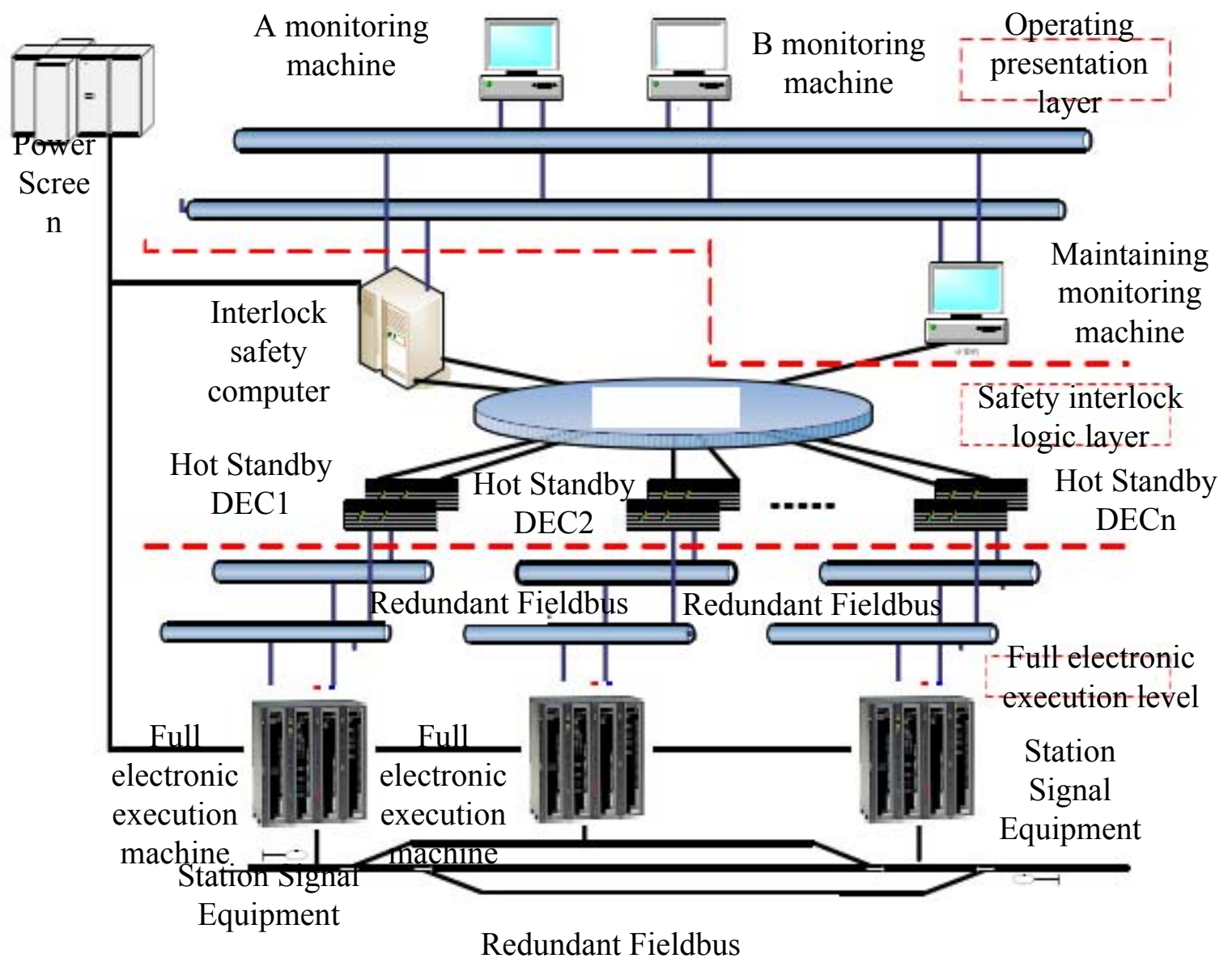

Diagram 1 Structure diagram of full electronic computer interlock system 


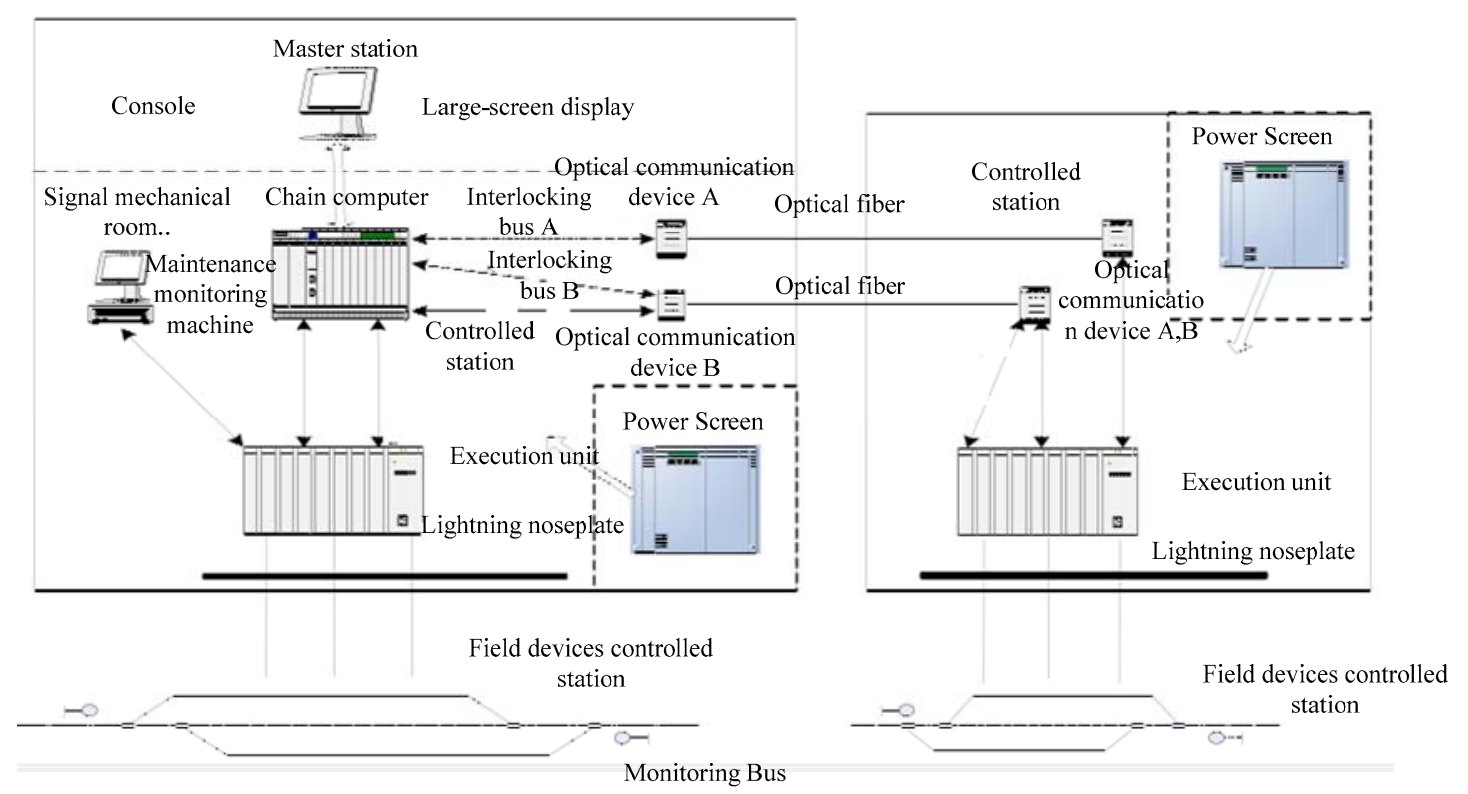

Diagram 2 Topology structure of regional control

As it is indicated by diagram 2, regional interlock control model can realize centralized control on signal equipment of many stations, but if the master station is paralyzed or there is network fault between master station and controlled station, which will cause station in regions can not normally manage railway operation task and affect efficiency shortage ${ }^{[6]}$ of railway transportation. Centralized control model is to set computer interlock control system in each station on the basis of interlock control model based on regional interlock control, monitoring computer in master station transmits operation command to each controlled station by redundant optical communication network, interlock system in controlled station implements operation command and feeds back state information in this station to master station for centralized display, realize centralized control by station signal equipment in station region as well as regional control model. When there is network fault between master station and controlled station or human authority, system is working under distributed model, operator can realize local interlock control operation in controlled station, as well as realize integrated control model of single station distribution model and multi-station centralized control.

\section{Railway signal control design based on intelligent learning algorithm}

It uses tree diagram as analysis tool, of which the non-end point represents decision problem, branch represents optional plan, it is one usual method for risk analysis and decision. It is based on theory of probability theory, its decision uses expectation value as standard, makes pruning and optimization by comparing with expectation value of each plan. Decision tree can intuitively reflect process of decision problem in different stage, especially for the complicated multi-stage decision, such as fault diagnosis of railway signal, stage indication, clear layer, which is favorable to make correct judgment. C4.5 can better deal with nature of continuous value and incomplete data, as well introduces into pruning technology, which simplifies decision tree and enhances accuracy rate and calculation efficiency of classification.

The information expectation I of calculating training set classification, I indicates dividing data training set into k kinds of degree with uncertainty. Divide training set $\mathrm{S}$ into k kinds, that is $\mathrm{S} 1, \mathrm{~S} 2, \ldots$, Sk. Suppose $\mathrm{d}$ is the observation sum of data concentration, di is the observation number of $\mathrm{Si}$, then probability belongs to $\mathrm{i}$ kind is $\mathrm{pi}=\mathrm{d} / \mathrm{d}(\mathrm{i}=1,2, \ldots, \mathrm{k})$, while information expectation of training set classification is as follows:

$$
I=-\sum_{i=1}^{k} p_{i} \log _{2}\left(P_{i}\right)
$$


Suppose A the first nature of training set, sj is observation number on $A=a j$, sij is the observation number corresponds to subset $\mathrm{Sj}$ on $\mathrm{A}=\mathrm{aj}$, while condition probability of certain example belongs to the $\mathrm{i}$ kind is $\mathrm{pij} \bullet \mathrm{pij}=\mathrm{sij} / \mathrm{sj}$, then information expectation of each value is as follows:

$$
I\left(A=a_{j}\right)=-\sum_{i=1}^{k} p_{i} \log _{2}\left(P_{i j}\right)
$$

Each possible value of node nature all corresponds to each subset, when observation data takes the same value in classification nature, and then generates decision tree. Root node starts, calculates false positive after pruning for each non-tree node, makes pruning for decision tree. Give one obvious level degree $=0.25$, the total number of error submits to distribution of 2 items:

$$
P\left[\frac{\left|p-p^{e}\right|}{\sqrt{p^{e}\left(1-p^{e}\right) / N}}>u_{1-\alpha}\right]=\alpha
$$

Of which, $\mathrm{p}=\mathrm{E} / \mathrm{N}$ is the false positive that really observed, $\mathrm{N}$ is the total number of tree after pruning, $E$ is the example number of appeared error after pruning. Suppose $z=1-\mu 1-\alpha$, take upper limit of region to estimate false positive, then calculation formula on false positive is as follows:

$$
p^{e}=\frac{p+\frac{z^{2}}{2 N}=z \sqrt{\frac{P}{N}-\frac{P^{2}}{N}+\frac{z^{2}}{4 N^{2}}}}{1+\frac{z^{2}}{N}}
$$

\section{Fault diagnosis expert system of railwaly signal equipment}

Fault diagnosis expert system of railway signal equipment is to use equipment state information obtained by micro-computer as diagnosis base and confirm position and cause of equipment by methods knowledge process, as well as give maintenance and decision system. Structure [8] of expert system is indicated by diagram 3 .

The work process of diagnosis expert system on signal equipment fault is as follows: firstly, micro-computer monitoring system makes real-time monitoring on operation state of equipment, data preprocessing module converts the collected original data into readable table data and adds to data base. Afterwards, it transmits to fault diagnosis module (including human interface, knowledge base, inference engine, explanation mechanism, data base and knowledge maintenance etc) by information interface. It will make matching on rules in data and knowledge base in advance according to expression transmitted by input symptom module, at the same time it obtains the other required information by human interface and personnel interaction, and then makes fault diagnosis. Finally, fault diagnosis expert gives prompt message on repair scheme; maintenance management module will realize dispatch of assignment so as to guarantee that fault can be timely dealt with.

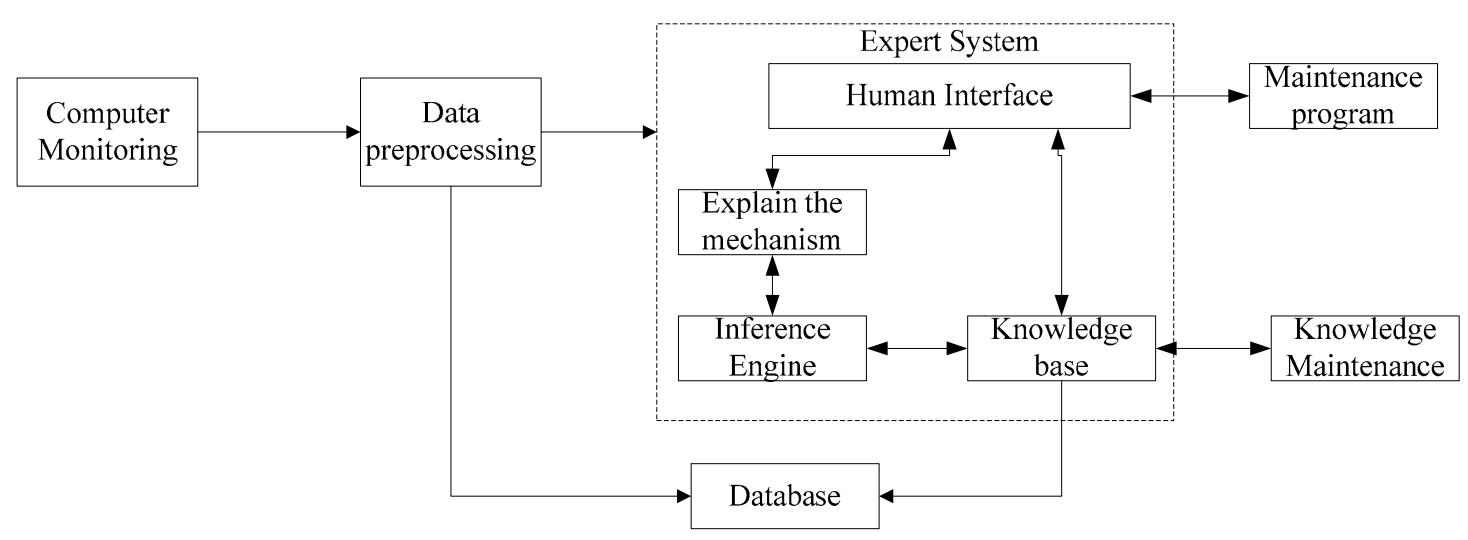

Diagram 3 Structure of expert system 
The diagnosis process on signal equipment fault is as follows:

a. Use expert system on fault diagnosis.

b. Make comparison on state information of equipment fault with data in fault characteristic vase, check matching record. If it includes record of this fault characteristic, add relevant rule to activation rule list of data base.

c. Choose one activation rule, compare with precondition of each rule. If precondition of certain rule is unknown, then consult users to contain more information.

d. If the all preconditions of this rule is known, then implement this rule and dispatch repair suggestion into data base.

e. Check whether there is other rules in activation rule list, if it has then repeat the above step $\mathrm{d}$ and $\mathrm{e}$.

$\mathrm{f}$. The repair suggestion of outputting matching degree is regarded as the final repair plan, if it has not obtained matching record, then output has no repair suggestion.

g. The repair suggestion of outputting matching degree is regarded as the final repair plan, if it has not obtained matching record, then output has no repair suggestion.

h. Fault diagnosis is completed.

\section{Knowledge presentation based on generated rule}

F (condition), THEN (conclusion) condition is also regarded as precondition or antecedent, it is used as condition whether this generated formula is usable or not. Conclusion is also regarded as consequent or action; it is the obtained conclusion or implemented operation after preconditions are met. Precondition can have many ways, each other combines with each other to form representation formula by logic calculation AND or OR. For example, the relay circuit of out of going out of station and button are indicated by diagram 4 .

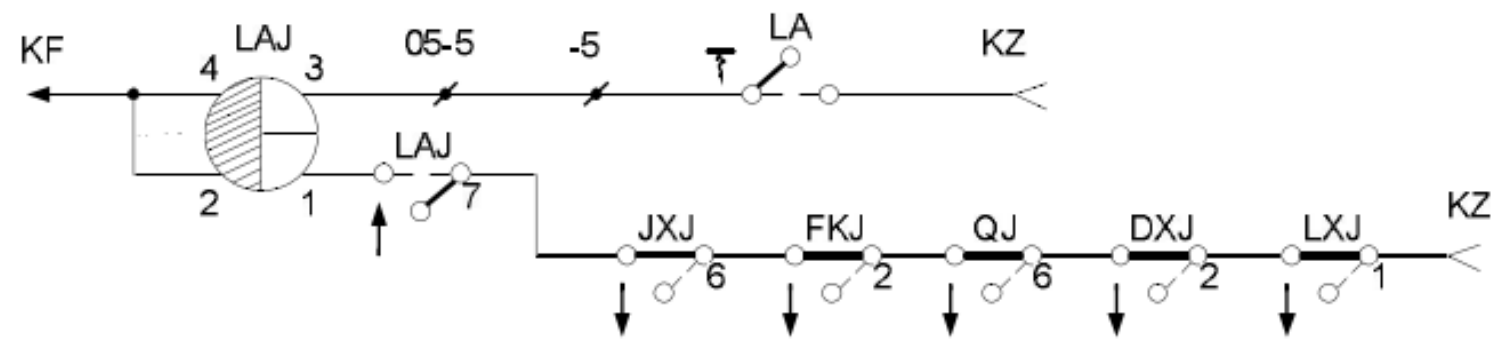

Rule 1:

Diagram 4 Relay circuit of going out of station and dispatch button

if indication lamp of access is not glittering.

and LAJ3-4 coil is power on

then conclude circuit fault is LAJ3-4 disconnected.

Rule 2:

if indication lamp of access button is not glittering.

and LAJ3-4 coil is power off.

and LAJ4 has no negative electricity.

then conclude circuit fault is disconnection in LAJ4 KF

The method of extracting knowledge from practicable experience is the hot point of research at present; it enables system to automatically collect knowledge by programming learning procedure. This kind of method has the ability of obtaining knowledge by itself, which means system can summarize new knowledge from operation of itself. Functions that self-learning mechanism should have are indicated by table 1 . 
Table 1 Function table of self-learning mechanism

\begin{tabular}{lll}
\hline Number & Function & Aim \\
\hline 1 & $\begin{array}{l}\text { language, word and image } \\
\text { identification } \\
\text { comprehension, analysis and } \\
\text { summary ability } \\
\text { check the completeness and } \\
\text { persistence of knowledge }\end{array}$ & $\begin{array}{l}\text { directly obtain original knowledge required by expert } \\
\text { system } \\
\text { summarize new knowledge from example and timely } \\
\text { report when it finds out conflict. } \\
\text { guarantee effectiveness of new knowledge }\end{array}$ \\
\end{tabular}

It is generated into new decision tree by learning algorithm of $\mathrm{C} 4.5$ and converts into the new rule Rule 3:

if indication lamp of access button is not glittering

and LAJ3-4 coil is power off

and LAJ4 has negative electricity

and combined side terminal $05-5$ is positive electricity

then conclude circuit fault is disconnection among LAJ3 05-5。

Rule order uses from of Rule * to represent the following: diagnosis result base is used to store description on expert diagnosis system and process suggestion of fault, then rule conclusion, information and reality are represented by way of Con*. Rule is used to establish relations between fault diagnosis base and result base of diagnosis.

\section{Realization on fault diagnosis based on decision tree $\mathbf{C} 4.5$ algorithm}

The diagnosis result base in knowledge base of expert system stores the detailed reality information in real circuit; it stores the repair suggestion given by expert on this reality information. Coverage rate of fault detection will directly affect fault diagnosis ability of this system, substitute different $\mathrm{C}$ values into Matlab to calculate its effect on system reliability and safety. Suppose each part all normally works in the beginning, the there is non-stop maintenance when system is continually working without stop, failure rate of system $/ 1=0.0001 / \mathrm{h}$, simulation time of safety $\mathrm{T}$ is $10000(\mathrm{~h})$, simulation time of reliability $\mathrm{T}$ is $10000(\mathrm{~h})$, , c value is respectively $0.7,0.8$ and 0.9 . The relations between safety and reliability are indicated by diagram 5 and 6 .
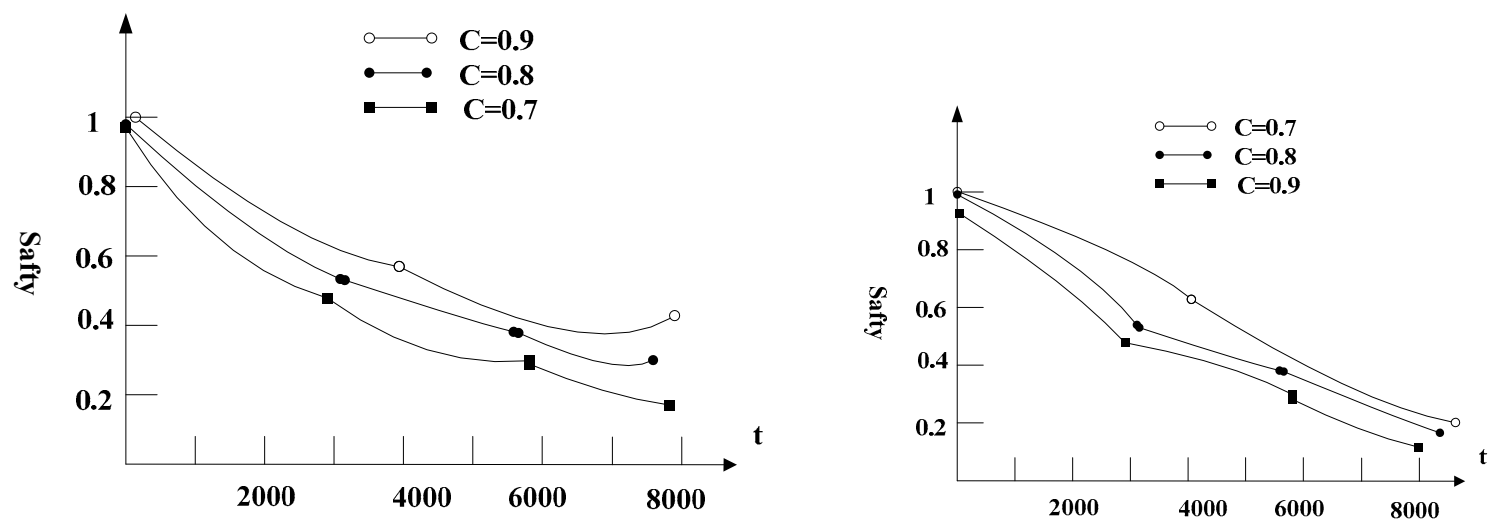

Diagram 5 Relations between system safety and c Diagram 6 Relations between system reliability and $\mathrm{c}$

From diagram 5 and 6 , we can see that coverage rate $\mathrm{C}$ of fault detection all has influence on system safety and reliability, system safety will obviously increase with increase of $\mathrm{C}$, while system reliability will have increase of smaller degree with increase of $C$. Change of $C$ value has strong effect on safety, the change degree of safety curve is larger than that of reliability. It is suggested to coverage rate of fault detection take $\mathrm{c}=0.9$, and makes maintenance for system every 4000 hours, so complication of system hardware is relatively proper, at the same time, reliability and safety can also keep higher level. 


\section{Summary}

Learning algorithm of $\mathrm{C} 4.5$ has characteristics of self-learning and incremental learning, which can realize automatic achievement on knowledge; it better solves the difficulty for knowledge acquisition. In addition, this algorithm uses decision tree synthesizes knowledge presentation and knowledge acquisition all in one, making knowledge presentation and acquisition in progress at the same time, it increases reasoning efficiency, provides theory base for equipment fault diagnosis used in railway signal. Through simulation and analysis on example, it demonstrates effectiveness of C4.5 learning algorithm used in fault diagnosis of railway signal equipment. Learning algorithm can make improvement according to actual fault, or it can obtain better result, the detailed classification and maintenance of fault need experience and suggestion of experts to confirm, the maintenance on knowledge base mainly relies on manual input etc at present.

\section{References}

[1] Zhao Zhixi.Control System on Station Signal. China Railway Publishing House,1995, p71-82.

[2] Lv Yongchang, Lin Yujun. Computer Interlock. China Railway Publishing House, 2007., p59-66.

[3] He Tao, Fan Duowang, Wei Zongtao, Fang Yafei . Research on Full Electronic Implementation Unit of Computer Interlock in Railway Station. Railway Journal ,2007, p184-192..

[4] Lv Jidong, Tang Tao, Yan Fei, Xu Tianhua. Modeling and Demonstration on Control Sub-system of CBTC Region Based on City Railway Traffic of UPPAAL. Railway Jounal.2009, p51-62.

[5] Xiang Yan,Xinya Sun,Yindong Ji.The modeling and simulation of high-speed railway signal Equipment based on component technology.2009 ICCSIT.PP:73-77.

[6] Hyun-Jeong Jo,Jong-Gyu Hwang,et al.Formal requirements specification in safety-critical railway signaling system.TD-ASIA.2009.pp:1-4. . .

[7] Dobias R,Konarski J,et al.Dependability Evaluation of Real Railway Interlocking Device. DSD. 2008. pp: 228-233. .

[8] Ogunsola A.EMC and Functional Safety requirements - Railway signaling applications.EMC in Railways,2009 IET Semina.pp:1-8.

[9] Zhang Wei. Research on Fault Diagnosis on Micro-computer Monitoring System Based on Data Mining.Lanzhou: Lanzhou Jiaotong University, 2010. 28-29.

[10] Zhang Baoyin, Liang Chaohui, Li Yongyan. Research on Expert System of Fault Diagnosis in Railway Signal Equipment ,2010,9., p115-117. 